\title{
Mythos
}

MYTHOS

Rivista di Storia delle Religioni

$11 \mid 2017$

Varia

\section{Technai e prerogative divine sulla scena del teatro di Eschilo*}

Technai and Qualities of the Gods on the Stage of Aeschylus' Dramas

\section{Franco Giorgianni}

\section{(2) OpenEdition \\ 1 Journals}

Edizione digitale

URL: https://journals.openedition.org/mythos/642

DOI: $10.4000 /$ mythos. 642

ISSN: 2037-7746

\section{Editore}

Salvatore Sciascia Editore

\section{Edizione cartacea}

Data di pubblicazione: 1 décembre 2017

Paginazione: 131-144

ISSN: 1972-2516

\section{Notizia bibliografica digitale}

Franco Giorgianni, «Technai e prerogative divine sulla scena del teatro di Eschilo*», Mythos [Online], 11 । 2017, online dal 24 septembre 2019, consultato il 21 septembre 2021. URL: http:// journals.openedition.org/mythos/642; DOI: https://doi.org/10.4000/mythos.642 


\section{Technai e prerogative divine sulla scena del teatro di Eschilo*}

\section{Franco Giorgianni}

\begin{abstract}
Riassunto
Lo studio indaga la posizione delle technai nell'opera di Eschilo, con particolare attenzione per il ruolo occupato dalle divinità come originarie, quando non esclusive detentrici e inventrici dei saperi tecnici. Attraverso una dettagliata disamina dell'uso del vocabolario della techne nei diversi drammi eschilei, emerge una doppia accezione del sapere tecnico, sia tradizionale che specificamente prometeico. In tal senso, la vicenda drammatica descritta nel Prometeo incatenato appare particolarmente rilevante per cogliere motivi di continuità e differenze all'interno del sistema di pensiero greco, specificamente eschileo, sulla techne. Chiude la disamina una postilla critica su due passi eschilei ( $\mathrm{Ag}$. 1129; fr. 375 Radt).
\end{abstract}

\begin{abstract}
The paper investigates so-called technai in Aeschylus' works, particularly with regard to the role attributed to the Gods as primary, if not unique actors and discoverers of technical knowledge. From the detailed study of the vocabulary of the word techne and its connected forms, extended to all Aeschylean dramas, a double meaning of technical knowledge emerges, a traditional as well as a specifically Promethean one. From this point of view, the dramatic plot described in Prometheus Bound seems to be particularly relevant in order to understand aspects of continuity and differences which underlie Greek thought and specifically Aeschylus' on techne. The paper closes by a critical review of the following two passages of the Aeschylus' work: Ag. v. 1129 and fr. 375 Radt.
\end{abstract}

\section{Parole chiave}

Technai • Prometeo - Eschilo - teatro e pensiero greco antico - prerogative divine e umane

\section{Keywords}

Technai • Prometheus • Aeschylus • ancient Greek drama and thought • divine and human qualities

\section{Premessa}

uesto studio nasce dalle ricerche da me condotte sul ruolo delle technai nel Prometeo incatenato di Eschilo ${ }^{1}$, e più in generale sull'uso del lessico connesso alla famiglia di techne nell 'ntera produzione del drammaturgo ateniese. Partendo dal presupposto che la produzione eschilea rappresenta una chiave di lettura importante per comprendere l'uso e il rilievo culturale ed epistemico assunti dal sapere tecnico nell'Atene del V secolo, e che un ruolo decisivo nello sviluppo dell'idea greca di conoscenza e di progresso assume la concezione delle relazioni che legano dèi e uomini rispetto al possesso dei saperi definiti dalle technai, nelle pagine seguenti intendo mostrare che è possibile riconoscere una linea di pensiero, antropologico e storico-religioso, coerente, che attraversa l'intera produzione teatrale eschilea circa il possesso di prerogative tecniche da parte delle divinità, e per ciò che ne consegue rispetto all'uso che di esse fanno gli esseri umani.

Conformemente all'estensione epistemica e semantica assunta dal lessico della techne nell'ambito del Prometeo incatenato rispetto alla restante produzione eschilea, la struttura dell'articolo prevede a) una parte introduttiva in cui la nozione di techne appare sostanzialmente limitata al possesso divino della sapienza mantica; segue b) la sezione centrale dello studio,

* Ringrazio l'amico e collega Andrea Cozzo per l'incoraggiamento a realizzare questo articolo.

1 Mi riferisco in primo luogo alla traduzione italiana, curata insieme con il collega Nicola Cusumano, del Prometeo genetista di Claude Calame 2016. 
dedicata alle conseguenze del dono prometeico delle arti tecniche ai mortali, sia in termini di rapporti tra immortali e mortali, sia per quanto concerne la concezione e la valutazione delle rispettive prerogative e possibilità. L'indagine si chiude con c) il riesame critico di due passi eschilei (Ag. 1129; fr. 375 Radt) su cui più forte si è avvertita la tendenza della filologia congetturale a interpretare in malam partem il lessico della techne, rispetto invece ad un uso eschileo che si rivela complessivamente ancora abbastanza neutro dal punto di vista semantico.

\section{La somma arte che non mente: la mantica ( $\mu \alpha \nu \tau \mathrm{\tau} \kappa \eta \dot{)})$}

Un filo rosso lega l'intera produzione teatrale eschilea in termini di riconoscimento e valore tradizionali tributati ad una specifica arte, per la posizione di rilievo riconosciuta alla cosiddetta mantica, l'arte del vaticinio. Essa si contraddistingue innanzitutto per il suo statuto

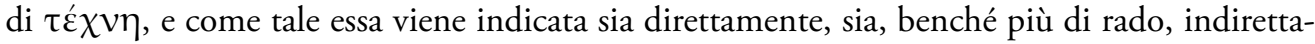
mente attraverso l'uso dell'aggettivo sostantivato $\mu \alpha v \tau \iota x \eta^{2}$. Detentori dell'arte mantica sono in primo luogo gli dèi, e in particolare Apollo e Zeus, anche se in effetti, alla luce di quanto detto dalla sacerdotessa Pizia in Eum. 17, Apollo è a sua volta "solo" un tramite della sapienza mantica di Zeus. Apollo, infatti, siede sul trono di Delfi come quarto e ultimo di una antichissima serie di profeti, che ha avuto inizio con la stessa Gea. Ad instillargli mente ispirata di arte profetica sarebbe stato appunto, a sentire la profetessa, Zeus in persona ${ }^{3}$. Dal punto di vista storico-religioso, quindi, considerando nel complesso tutte le occorrenze eschilee, l'arte mantica esercitata nel santuario delfico, con la sua sapienza e il suo linguaggio spesso ambiguo e verboso (vedi l'aggettivo $\pi \mathrm{o} \lambda v \varepsilon \pi \varepsilon \tilde{\iota} \varsigma$ riferito alle arti degli indovini in $A g .1134$ ), sembra rientrare più nella sfera d'influenza del potente Zeus, garante del più recente ordinamento cosmico e padre delle generazioni umane e divine, che in quella del pur tradizionalmente saggio Apollo. Ciò non toglie che gli esseri umani dotati di arte profetica, e quindi capaci di interpretare il volere degli dèi mettendoli così in comunicazione con i mortali, siano comunemente debitori della loro dote ad Apollo. Così, possesso umano dell'arte mantica e possessione da parte della divinità delfica finiscono per confondersi inscindibilmente nella personalità alienata di Cassandra, le cui parole, dai tristi esiti, ma non meno veritiere, sono destinate ad incontrare l'aperta resistenza degli interlocutori, dal primo momento in cui, per riprendere l'espressione del Coro dell'Agamennone, la giovane profetessa era già posseduta dalle arti profetiche che il dio ispira ( $\mathrm{Ag}$.

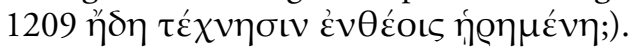

La mantica, nella rappresentazione che ne dànno i drammi di Eschilo, si presenta come un'arte assolutamente veritiera, che non mente (Sept. 26 ỏ $\psi \varepsilon v \delta \varepsilon \hat{\imath} \tau \dot{\varepsilon} \chi \nu \eta)$ e che è non senza effetto (Ag.

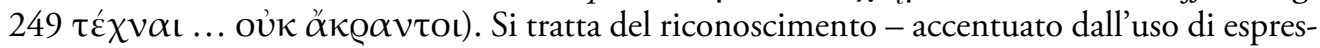
sioni litotiche - dell'ineluttabilità della volontà dei numi, ma in primo luogo dell'esistenza di un canale ermeneutico preferenziale tra uomini e dèi che passa attraverso la comprensione

2 Uno spoglio completo delle occorrenze relative mostra che il termine $\tau \dot{\varepsilon} \chi \nu \eta$ è usato in riferimento alla mantica nei seguenti passi: A. Eum. 17; Sept. 26; Prom. 497 (al singolare); Ag. 249, 1134, 1209 (al plurale), mentre l'uso

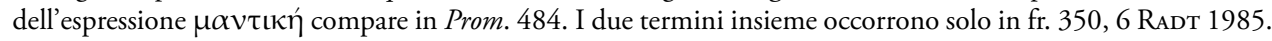
Si deve a CALAME 2016 la sottolineatura del carattere tecnico della mantica per il pensiero greco; di recente, lo stesso CALAME 2017, 393-397, è ritornato sull'argomento con un dettagliato riesame in chiave epistemica delle varie forme di divinazione descritte nel Prometeo incatenato, con l'intento di neutralizzare l'opposizione tradizionale tra divinazione e razionalità greca, che ha avuto uno dei sostenitori più autorevoli in Jean-Pierre Vernant.

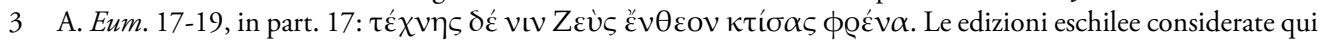
di riferimento sono West 1990; PAGE 1972. 
esclusiva da parte dell'indovino degli enigmi sentenziati dalla divinità. Dal punto di vista tecnico, entrando nel dettaglio della descrizione degli strumenti di cui il vate tebano Tiresia si serve per presagire il destino di sventura del casato di Edipo, il procedimento consiste nel fare appello ai propri orecchi e mente per comprendere i fruscii provenienti dal volo degli uccelli

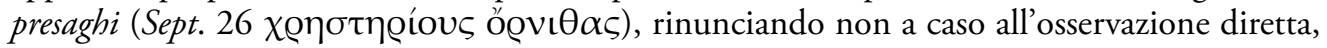
perché per un'antica colpa a Tiresia la vista è ormai preclusa. Si tratta di una mantica augurale in cui manca completamente l'uso del fuoco (Sept. $25 \pi$ voò Sí $\chi \alpha$ ), il che è in significativo contrasto con l'importanza che il fuoco assume nella pratica mantica non tradizionale (quella

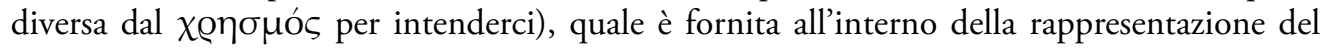
Prometeo incatenato ${ }^{4}$, dove al contrario, come vedremo in dettaglio più avanti, si attribuisce al fuoco programmaticamente un ruolo euristico di primo piano nella invenzione o rivelazione agli uomini delle technai in generale.

Sul piano della sua caratterizzazione antropologica, la mantica si configura, alla luce di quanto esposto sinora, come un'arte che solo gli interpreti di professione sono in grado di usare, e di cui gli uomini non sono i veri possessori, avendo ricevuto in dono un sapere che ha ancora tutte le caratteristiche di una prerogativa divina. L'attribuzione pressoché esclusiva alle divinità di questa specifica prerogativa tecnica si inserisce, del resto, nel sistema di pensiero greco tradizionale, in cui gli dei sono considerati gli "inventori” delle arti tecniche, che pertanto si presentano già perfette e compiute sin dal loro primo apparire, prive di una qualsiasi dimensione di sviluppo storico 5 . Da questo punto di vista, il teatro eschileo aderisce ad un siffatto orizzonte intellettuale, pur con delle interessanti peculiarità. Innanzitutto, va notato il ruolo assolutamente preminente giocato dalla mantica come techne nell'intera opera eschilea superstite, Prometeo incatenato incluso. Questa sottolineatura tecnica della mantica non può essere dovuta al caso, anzi probabilmente corrisponde ad un'idea tradizionale di techne fortemente ancorata al sapere divino, che si qualifica come vero sapere, specie nella sua declinazione delfico-apollinea. Del resto, come avrò modo di osservare nelle pagine seguenti, la caratterizzazione dei personaggi del Prometeo incatenato non sfugge del tutto a questa prospettiva, e ciò trova conferma anche nella struttura drammatica della stessa tragedia, fondamentalmente incentrata sulle conseguenze nefaste per Prometeo e per l'intera umanità della risolutezza del Titano a non mettere al servizio di Zeus la propria sapienza profetica, rivelando quale futura unione matrimoniale comporterà la fine del suo potere.

A ciò si aggiunga che la mantica è indicata come arte per eccellenza che non falla (Sept. 26 $\dot{\alpha} \psi \varepsilon v \delta \eta ́ \varsigma)$. L'infallibilità della mantica si traduce per i mortali nell'attesa di un ineluttabile destino di sventura, o almeno così si esprime il Coro dell'Agamennone di fronte alle funeste rivelazioni di Cassandra, dando voce probabilmente al carico di angosce che per la gente comune

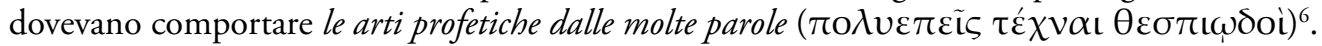
Gli stessi dei ripongono assoluta fiducia nelle parole che promanano dalla veritiera bocca del

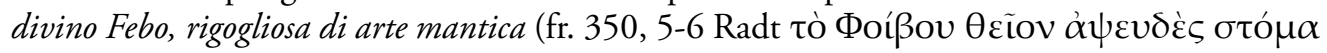

4 Le varie forme della mantica squadernate agli uomini da Prometeo sono descritte in Prom. $484 \mathrm{sgg}$.

5 A questo proposito vedi le osservazioni di Cambiano 1991, 16-18 sulla posizione critica di Senofane nei confronti della tradizione; al ruolo di Senofane per l'affermazione dell'idea di progresso attribuisce un forte peso EDELSTEIN 1987, 55 sgg.

6 Ag. 1132-1135: la parola profetica comporta per i mortali aspettativa di sventura e apprendimento di paura

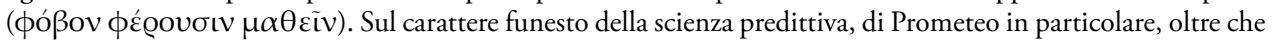
di Apollo, vedi l'ampia disamina in SAÏD 1985, 197 sgg. 


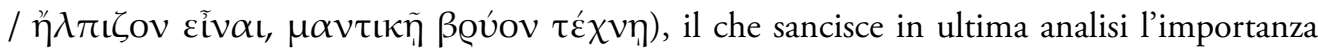
e l'utilità della divinazione non solo per gli uomini, ma anche per gli dèi. Entrambi, infatti, contrariamente a ciò che si potrebbe pensare, sono soggetti al compiersi ineluttabile del destino, e nella mentalità che accomuna Eschilo ad altri intellettuali dell'età arcaica, tra cui Esiodo, Solone e Pindaro, più che Zeus, sono divinità antichissime e perciò venerande come Gea-Temis, le Moire triformi e le memori Erinni a reggere le fila del destino ${ }^{7}$. Infatti, su tutto e tutti, nonostante gli sforzi di uomini e dèi per volgere il corso degli eventi a proprio favore, domina necessità ( $\dot{\alpha} v \alpha \dot{\gamma} \gamma \kappa \eta)$.

Si può dire in effetti che lo statuto tecnico della mantica viene affermato nell'intera produzione drammatica di Eschilo, e la vicenda del Prometeo incatenato non rappresenta in tal senso un'eccezione, anzi in quest'ultimo dramma il valore strategico della divinazione nella lotta per il potere tra le generazioni divine (Zeus e gli Olimpii da una parte, i Titani ribelli dall'altra) viene addirittura esaltato. C'è però, pur nel segno della continuità, una sensibile differenza tra la concezione della mantica come è presentata nel Prometeo rispetto agli altri drammi eschilei: sul piano delle prerogative divine, essa rimane un dono di un dio, nella fattispecie del Titano, ai mortali, ma la sua caratterizzazione come techne si arricchisce di una dimensione praticoutilitaristica sinora sconosciuta ${ }^{8}$. Al pari di altre tecniche prometeiche, la mantica è un poros, ossia uno strumento che il Titano offre ai mortali per la comprensione del mondo, e quindi essa assume un carattere propriamente semiotico, come è stato già sottolineato in studi precedenti'. Non è più soltanto l'espressione di un sapere oscuro e di difficile interpretazione

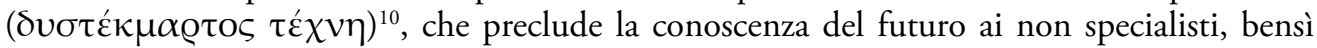
diventa un'arte prognostica che, al pari della medicina ${ }^{11}$, illumina della propria conoscenza le vie del futuro e consente a chi la possiede di avvalersene a proprio vantaggio, sia uomo o dio.

\section{Technai tradizionali e arti prometeiche nel Prometeo incatenato}

Con la rappresentazione della trilogia dedicata al mito prometeico ${ }^{12}$, Eschilo si confronta

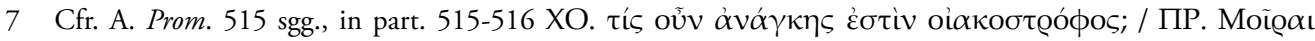

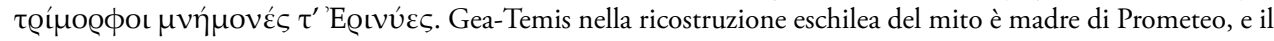
Titano ha ereditato da lei la capacità profetica.

8 Sul carattere laico della mantica come appare nell'Incatenato, vedi Calame 2017, 397-399.

9 Di una sapienza di tipo pratico-conoscitivo parla Di BENEDETTO I978, 99 con n. 12; l'aspetto semiotico delle technai nell'insegnamento che il Titano ne fa agli uomini è stato sottolineato da Calame 2016, 31-35.

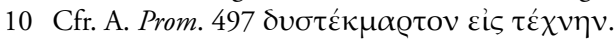

11 I confronti con l'arte medica e le metafore mediche per descrivere la condizione di Prometeo ricorrono con una certa frequenza nell'Incatenato, vedi per tutti Prom. 472-475, versi in cui il Coro interrompe la lunga tirata di Prometeo sui benefici all'umanità attribuendogli una condizione patologica cui non sa porre rimedio, al pari di un cattivo medico che si è ammalato e non sa curarsi (una lunga discussione sulla tematica è presente in SAïD 1985, 169-186). Sulle analogie di metodo e di linguaggio scientifico tra divinazione e prognosi medica vedi Ead., 192-195.

12 Problematica appare soprattutto la posizione del dramma intitolato Prometeo portatore di fuoco (Поo

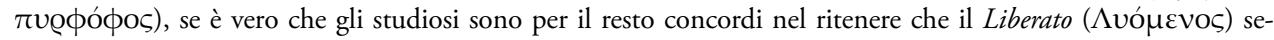
guisse l'Incatenato $(\Delta \varepsilon \sigma \mu \omega \tau \eta\rceil)$, cfr. RADT 1985, 329. Quanto alla vexata quaestio del carattere pseudoepigrafo dell'Incatenato, sostenuto tra gli altri da West, Griffith e Marzullo, gli studi della SAïD 1985, part. 79 sg., e della PatToni 1987, che ha sottoposto ad un'attentissima disamina la struttura sia metrico-stilistica che drammatica della tragedia, pervengono alla conclusione che nulla osta a che si possa ritenere valida l'attribuzione tradizionale ad Eschilo del dramma. Già Dı BENEDETTO 1978 ne aveva argomentato ampiamente l'autenticità, soffermandosi tra gli altri dettagli sulla coerenza dei tratti assunti dal carattere di Zeus, ovvero del valore assunto dalla terminologia inerente la sapienza, con la concezione eschilea. CALAME 2016, 31, lascia aperta la questione dell'autenticità. 
con la necessità di tenere conto di un vasto numero di saperi che rientravano ormai a pieno titolo nel novero delle cosiddette arti tecniche, le $\tau \dot{\varepsilon} \chi \nu \alpha$ เ. In effetti, al tradizionale ruolo di Prometeo, che insieme con Atena e Efesto godeva di un particolare culto tra gli artigiani di Atene, quale astuto oppositore di Zeus e benefattore del genere umano, Eschilo affianca quello di eroe civilizzatore, opposto a Zeus dalla volontà di proteggere gli umani dalla distruzione cui sarebbero stati votati, ove avesse prevalso il volere del padre degli dèi di escludere gli uomini dalla distribuzione delle originarie prerogative. La scelta del drammaturgo ateniese consente così di inserire la vicenda di Prometeo nel solco di una tradizione secolare che collegava il furto del fuoco e le astuzie prometeiche al momento fondativo di attività umane essenziali come il lavoro, specialmente agricolo, e l'istituzione del sacrificio, grazie a cui l'uomo, pur separandosi irrimediabilmente dal consesso divino, recupera quel bios che gli dèi della generazione olimpica gli avevano sottratto ${ }^{13}$.

Il dono prometeico delle technai agli uomini, pur riaffermando la teoria dell'origine divina delle arti e dei saperi tecnici, espone l'essere umano ad una duplice sfida: da una parte, gli consente di spostare in avanti il limite della propria mortalità, con l'opportunità non solo di sopravvivere ma di vivere a proprio agio orientandosi nella realtà che lo circonda, e dall'altra lo mette a confronto coi dilemmi etici e sociali posti dal possesso della techne.

Da questo punto di vista, uno studio delle occorrenze dei termini connessi con la radice di techne all'interno del Prometeo incatenato consente, a mio parere, di misurare la distanza non solo semantica, ma soprattutto culturale e gnoseologica che separa un certo uso delle technai, di stampo direi tradizionale, dalla nuova accezione prometeica che esse sembrano assumere. La declinazione prometeica imprime alle technai una svolta decisiva verso un'appropriazione umana dei saperi coinvolti; dal punto di vista drammatico, ciò si traduce in un forte coinvolgimento emotivo del pubblico, che da un lato è indotto a simpatizzare e a provare umana comprensione per le sofferenze del filantropico Prometeo ${ }^{14}$, "ingiustamente" punito e umiliato da un potere tanto recente quanto dispotico e per ciò stesso violento, quello di Zeus, ma dall'altro impara quanto può costare caro opporsi in maniera sprezzante alla volontà di Zeus, dopo avere rubato agli dèi per donarle ai mortali le arti derivanti dal fuoco. In termini antropologici, se è vero che i pathe di Prometeo e la sua esperienza di sofferenza richiedono la partecipazione del pubblico, è pure vero che essi chiamano direttamente in causa la responsabilità dell'agire umano, pongono l'essere umano a confronto con il senso di vertigine che deriva dal mettere mano a cose divine, le technai specificamente. In questo modo, a mio parere, dal punto di vista della concezione greca del tempo, finiscono per convivere sulla scena del teatro eschileo due diverse nozioni di techne: l'una proiettata ex abrupto sullo sfondo della storia umana, grazie alla quale

Una traduzione completa dei frammenti della trilogia propongono G. e M. Morani 1987, 689-695.

13 La dimensione agricola, e quindi pre-artigianale, del lavoro nella versione esiodea del mito prometeico è messa in evidenza da Vernant 1978, 273 sgg.; sul mito di Prometeo nelle sue differenti versioni da Esiodo a Platone, cfr. ora Calame 2016, 26-46. Sul nascondimento del bios umano da parte degli dèì cfr. Hes. Op. 42 sgg. Il sacrificio ha le sue radici etiologiche nella vicenda dell'inganno perpetrato da Prometeo ai danni degli dèi a Mecone, come narra Hes. Th. 535 sgg.

14 Tale coinvolgimento ha luogo durante tutto il corso della rappresentazione, già dall'apparire sulla scena di Efesto, che suo malgrado è chiamato a incatenare Prometeo, ma soprattutto grazie all'intervento del Coro delle ninfe Oceanine, per molti versi emotivamente complice, specie all'inizio, della vicenda del Titano, per toccare il suo apice al comparire sulla scena del personaggio della vergine Io, come Prometeo vittima della prepotenza di Zeus, ma a differenza di Prometeo, del tutto umana. 
gli uomini che prima vivevano come formiche negli antri della terra ${ }^{15}$, assumono ora tratti di civilizzazione, l'altra ancora schiacciata sull'unidimensionalità temporale di una sempiterna perfezione, tradizionalmente legata all'idea di un'intima coesione tra possesso di un'arte e in-

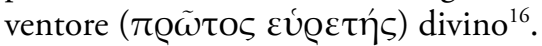

Espressione della techne più tradizionale, nella sua versione specificamente artigianale, è il dio Efesto, presentato come materiale esecutore dell'ordine impartito dalle alte sfere divine di legare, imprigionare, impedire con vincoli indissolubili colui che è capace di trovare una via di fuga dalle situazioni più difficili, appunto Prometeo ${ }^{17}$. Controllato a vista nell'esecuzione dell'opera dalla forza coercitiva del potere, rappresentata da Kratos e Bia, Efesto completa con la consueta perizia e senza sforzo la sua opera all'inizio del dramma; due sono le occorrenze del termine $\tau \dot{c} \chi v \eta$ che si riferiscono al suo operato, e sono utili a chiarire di quale arte è in possesso il dio artefice.

Sin dal suo apparire sulla scena, Efesto si contraddistingue per la sua vicinanza etica al Titano, anche in nome della comune discendenza da Urano; è il dio stesso che in uno scambio di battute con Kratos, definisce quale molto invisa $\chi \varepsilon\llcorner\varrho \omega v \alpha \xi i ́ \alpha$ la propria attività (Prom. 45), al che Kratos ribatte che all'arte di Efesto non si può imputare alcuna responsabilità della

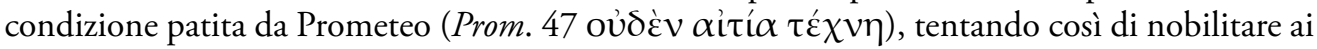
propri occhi e davanti al pubblico l'azione svolta da Efesto, definita nei modi tradizionali della inclita arte del dio artigiano. Da parte sua, Efesto stesso considera la propria una mera attività manuale al servizio di altri, e in quanto tale propriamente servile ${ }^{18}$, tanto che alla successiva battuta lamenta che questo ingrato compito non sia toccato ad altri (Prom. 48). A lui tocca di forgiare e assicurare i legami adamantini che impediscono i movimenti all'astuto Prometeo, in una gara che ad arte contrappone arte, alla sapienza astuta del Titano la perfetta abilità demiurgica di Efesto, che si presta solo suo malgrado agli inviti di Kratos di stringere, serrare e inchiodare il corpo del Titano alla roccia del Caucaso. Non è quindi un caso se alla fine delle operazioni di inchiodatura e saldatura realizzate da Efesto, Kratos si rivolge con tono sprezzante e provocatorio a Prometeo, esortandolo a trovare, lui che a torto chiamano "Previdente", un

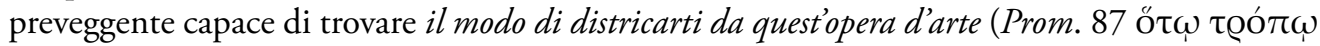

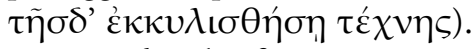

La techne di Efesto si caratterizza quindi come un'abilità artigianale tradizionale di origine divina, capacità di portare a compimento con sapienza un'opera d'arte, realizzata secondo i canoni, demiurgicamente prestata per conto di altri ${ }^{19}$, in questo caso al servizio della stringente necessità $(\dot{\alpha} \vee \alpha ́ \alpha \kappa \eta)^{20}$, e quindi non libera, bensì una vera e propria $\chi \varepsilon \iota \varrho \omega v \alpha \xi \hat{i} \alpha^{21}$.

In maniera del tutto diversa si caratterizza la techne di cui dispone Prometeo e che dona agli

15 Cfr. A. Prom. $452-453$ sotterranei vivevano come agili / formiche ( $\mu$ $\varrho \mu \eta \kappa \varepsilon \varsigma)$ in recessi di caverne non illuminati dal sole.

16 Cfr. Griffith 1983, 167.

17 Cfr. A. Prom. 59 (sono parole di Kratos ad Efesto) è capace di scovare una via d'uscita anche da situazioni

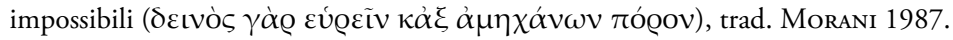

18 L'unica altra attestazione eschilea del termine $\chi \varepsilon\llcorner\varrho \omega v \alpha \xi i ́ \alpha$ occorre in Cho. 761, dove la nutrice di Oreste, all'an-

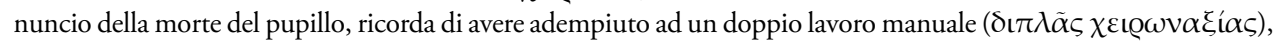

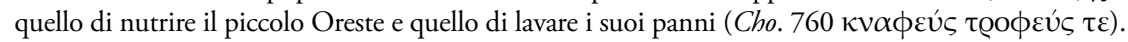

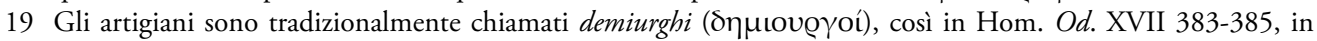
quanto prestano la propria opera al servizio e su richiesta del demos.

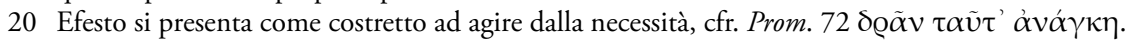

21 Sul significato del termine, inteso come Tätigkeit einer unfreien Bediensteten, vedi LöBL 1997, 77. 
uomini. Anche quando si tratta di un'attività artigianale - quale ad esempio la costruzione di case o la lavorazione del legno (Prom. 450-451) - la techne equivale nelle parole dello stesso Prometeo ad un espediente attraverso cui gli uomini sfuggono, o meglio sperano di sfuggire, alla propria condizione di mortali, è insomma un cosiddetto poros, una via di fuga dalle situazioni difficili. I due termini, arti tecniche e risorse, compaiono non casualmente in stretta rela-

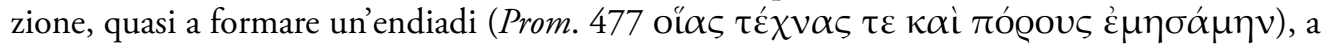
introdurre una lunga lista di escogitazioni inventate a vantaggio dell'umanità.

In effetti, quello che si può definire il catalogo delle invenzioni prometeiche ha inizio alcune decine di versi prima del passo testé citato, e si suddivide in due lunghe sezioni, esattamente corrispondenti tra loro per estensione: la prima comprende i versi 441-471, la seconda i versi 476-506, interrotte da poche battute del Coro (vv. 472-475). Per comprendere in maniera approfondita il senso culturale ed epistemico della nozione prometeica di techne, è utile riassumere dal punto di vista terminologico le espressioni che descrivono le invenzioni del Titano. Si tratta innanzitutto di benefici, di doni che Prometeo ha elargito agli uomini, per puro amore

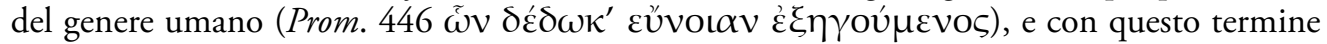
si possono riassumere a) genericamente tutti i benefici dell'intero catalogo (vv. 441-506), b) specificamente solo le invenzioni della prima parte del catalogo (vv. 441-471). Ciò implica che una serie di termini, dal carattere descrittivo eppure culturalmente significativi, delineano presi nel loro complesso il campo semantico e gnoseologico di riferimento delle technai prometeiche, e che d'altra parte i già citati technai e poroi concorrono a definire un tale ambito. Il primo ordine di benefici (vv. 447-453) attiene all'attivazione della sfera che potremmo chiamare percettivo-conoscitiva: gli uomini, prima della donazione prometeica, avevano sensazione, ma non vera percezione dei fenomeni e degli impulsi provenienti dall'ambiente circostante; vivevano a caso (Prom. 450 عikñ)), simili a forme di sogni per l'intera durata della vita (Prom. 448-

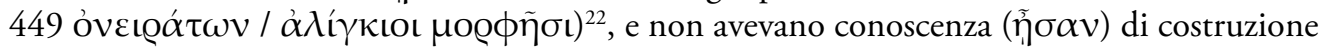
di case fatte di mattoni né di lavorazione del legno. Nel medesimo ambito conoscitivo sembrano rientrare le indicazioni fornite da Prometeo in relazione all'orientamento spazio-temporale (vv. 454-458), dal momento che i primi uomini non disponevano di conoscenze indiziarie

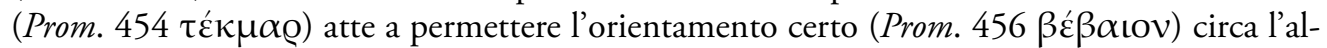
ternarsi delle stagioni, sicché levarsi e tramontare degli astri erano senza criterio (Prom. 458

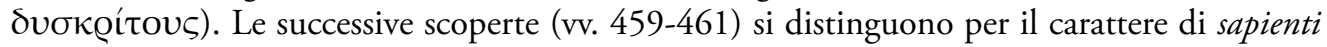
invenzioni ( $\sigma 0 \phi i ́ \sigma \mu \alpha \tau \alpha)$, ossia l'invenzione del sistema alfanumerico delle lettere dell'alfabeto, che vengono in aiuto alla memoria. Questa prima serie è chiusa dall'elenco (vv. 462-469)

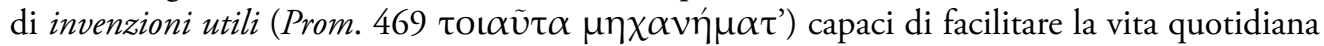
degli uomini, ossia l'aggiogamento delle bestie selvagge, la costruzione di carri e di navi. Le ultime due serie di invenzioni appena citate possiedono più un carattere pratico-utilitaristico, e in particolare l'uso delle lettere dell'alfabeto permette di fissare in maniera indelebile per iscritto la memoria di segni che altrimenti andrebbero perduti, se affidati alla mera oralità. Ricorre, in tutti questi versi della prima sezione, con particolare frequenza il lessico dell'invenzione/

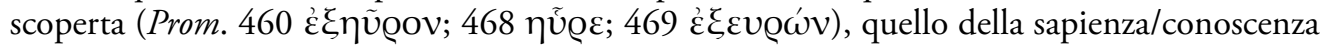

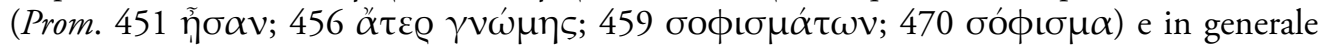

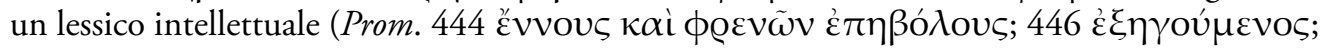

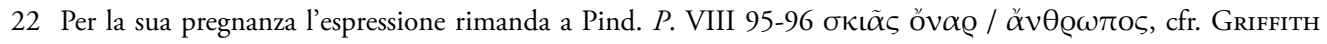
$1983,165 \mathrm{sg}$. 


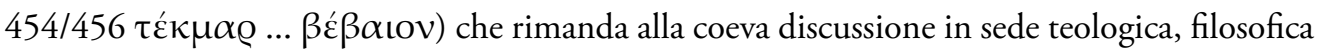
e sofistica ${ }^{23}$.

La seconda sezione del catalogo delle invenzioni prometeiche, cui avevo solo accennato poc'anzi, prende quindi l'avvio: si tratta di una risposta alle parole del Coro, critico nei confronti di Prometeo, e ha tutta l'aria di riprendere letteralmente, volgendolo a proprio favore - ma al contempo confermandone la sostanza - quanto sostenuto poco prima dalle Oceanine: Prometeo delirerebbe e si comporterebbe come un cattivo medico che non sa curare se stesso

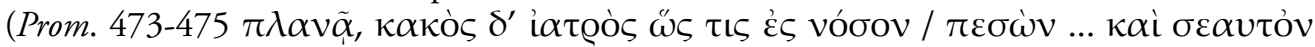

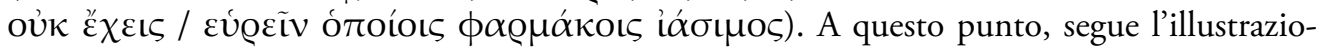
ne di quei ritrovati che Prometeo stesso qualifica come technai e poroi (vv. 476-506), senza che si possa peraltro escludere che questa espressione valga anche per le invenzioni che sono state nominate prima. Seguendo verosimilmente un'associazione di idee che ha le sue prime tracce nella poesia epica ${ }^{24}$, le prime due arti che Prometeo nomina sono la medicina (vv. 478-

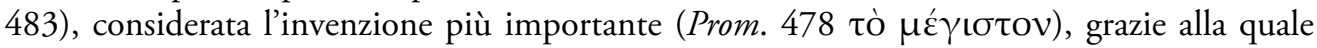

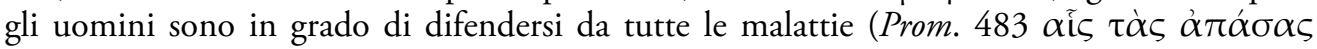

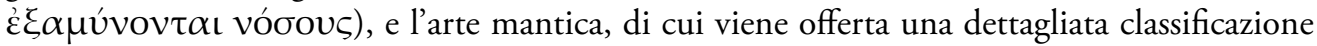
(vv. 484-499), che significativamente occupa il più ampio numero di versi tra le invenzioni in catalogo, quasi a sancire l'importanza che quest'arte occupa tradizionalmente nel contesto della produzione eschilea. La divinazione è distinta dapprima con il termine mantica (Prom.

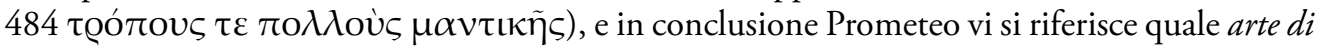
difficile interpretazione (Prom. $497 \delta v \sigma \tau \varepsilon \dot{\kappa} \mu \alpha \varrho \tau о \varsigma \tau \varepsilon ́ \chi \vee \eta)$, distinguendone appunto quattro

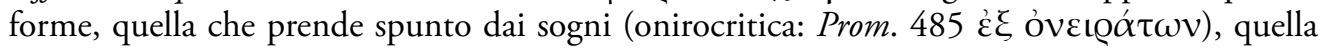
che si applica alle voci e ai segni che si incontrano per strada (vv. 486-487), la tecnica augurale che interpreta il volo degli uccelli (vv. 488-492), e quella che si basa sulla lettura delle viscere delle vittime (vv. 493-499). Nell'ultima parte del suo resoconto, Prometeo passa in rassegna

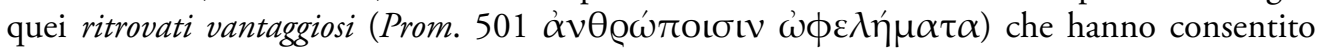
agli uomini di sfruttare i giacimenti di minerali nascosti nel sottosuolo.

Anche in questo caso prevale il lessico relativo al sapere e alle invenzioni, alle escogitazioni pratiche, questa volta con un'accezione propriamente semiotica, nel senso che Prometeo ha mostrato, messo a nudo, indicato e significato attraverso segni, indizi e altri indicatori visibili e percepibili dagli organi di senso e dall'intelligenza umana ambiti di esperienza sinora inesplorati $^{25}$. In effetti, è stato Prometeo il primo a mostrare (v. 482 है $\delta \varepsilon\llcorner\xi \alpha)$ le opportune mescolanze

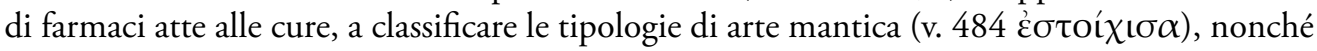
a rendere riconoscibili (v. $487 \dot{\varepsilon} \gamma \nu \omega ́$ Qı $\sigma \alpha)$ i criteri grazie a cui associare nell'interpretazione

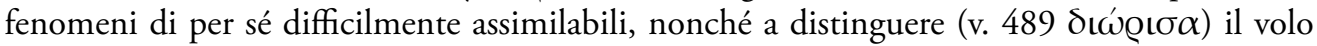

23 A questo proposito, si rinvia alla complessa e dettagliata disamina del problema in SAÏD 1985, 131-168, che tirando le somme tende piuttosto à opposer le Prométhée à la sophistique, ibid., 144. Ha ragionato sulle accezioni semantiche della famiglia lessicale connessa a ooфí $\alpha$ Di BenedeTto 1978, 94 sgg., individuando un'opposizione tra sapienza tradizionale e sapienza prometeica (in cui rientrano a pieno titolo i citati sophismata). Dà una lettura teologica del dramma e del ruolo svolto dalle technai Löвl 2003, $270 \mathrm{sg}$.

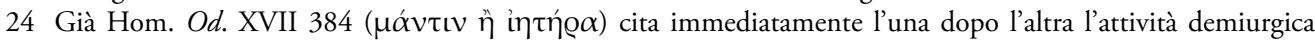
dell'indovino e del medico, e secondo la medesima sequenza occorre la menzione delle due figure anche in Sol. fr. 1 Gentili, Prato 1979, e in entrambi i casi la loro attività è posta dal poeta sotto l'egida di una specifica divinità: la mantica deriva all'indovino da Apollo (53 sgg.), laddove i medici eseguono l'opera di Peone (58 sgg.).

25 Sul valore semiotico delle technai prometeiche ha insistito soprattutto Calame 2916, 32-35. 
degli uccelli differenti, creandone una vera e propria tassonomia augurale, sino all'illustrazione (v. $499 \dot{\varepsilon} \xi \omega \mu \mu \alpha \dot{\tau} \omega \sigma \alpha$ ) dei segni, prima oscuri, che brillano alla luce del fuoco dei sacrifici

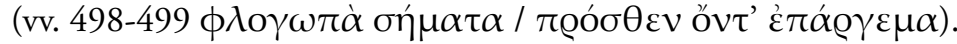

Sin qui gli aspetti euristici e positivamente prometeici delle technai inventate o reinventate dal Titano. Ma a ben vedere, si tratta di invenzioni e risorse pur sempre riconducibili ad un protos heuretès divino, Prometeo. A ciò si aggiunga che le technai di Prometeo, come si dice

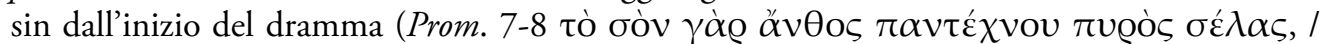

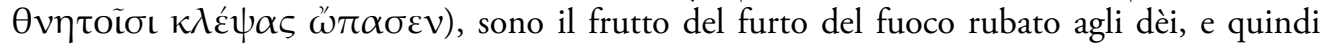
rappresentano nel loro applicarsi da parte dell'uomo un possibile atto di hybris. Esse sono state inventate per permettere un poros al genere umano nella sua interezza, ma hanno dei chiari limiti, nella misura in cui si tramutano in una trappola per lo stesso Prometeo, il quale, a suo stesso dire, non è in grado di trovare una mossa sapiente con cui trarsi d'impaccio (Prom.

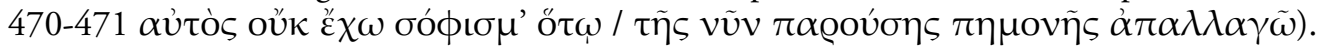
Nonostante tutte le sue proprie escogitazioni vengano ricondotte da Prometeo alla categoria dei vantaggi (v. $501 \omega \dot{\omega} \phi \varepsilon \lambda \eta ́ \mu \alpha \tau \alpha$ ) per il genere umano, si tratta di un miraggio euristico, giacché in effetti, a detta dello stesso Prometeo (Prom. 248 sgg.), il dono più significativo del Titano è consistito nell'impedire ai mortali di prevedere il proprio destino mortale (v. 248 $\pi \varrho 0 \delta \varepsilon ́(\kappa \varepsilon \sigma \theta \alpha \iota \mu$ uó@ov), ossia nello stornare il più possibile il giorno della morte, propinando il farmaco più adatto in tal senso, consistente nel fare albergare negli uomini cieche speranze (v. $250 \tau v \phi \lambda \grave{\alpha} \varsigma \dot{\varepsilon} \lambda \tau i ́ \delta \alpha \varsigma$ ). Quelle technai che nelle parole di Prometeo rappresentano delle possibilità in più per l'intero genere umano, nel gioco delle parti che prende forma sulla scena del dramma eschileo, si tramutano, nelle parole degli altri personaggi, in una folle sfida contro la necessità, contro il potere di Zeus, contro l'ineluttabile destino di morte degli uomini, tanto che il Coro può con lucida ironia definire proprio un gran giovamento quello di cui Prometeo

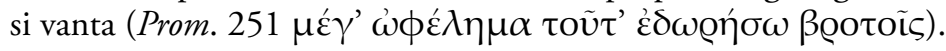

\section{3. "Tutte le arti provengono agli uomini da Prometeo"}

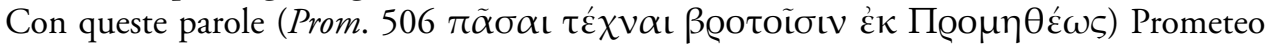
sigilla orgogliosamente la conclusione del lungo elenco delle invenzioni con cui hai beneficato i mortali. Così facendo, Prometeo avoca a sé la prima invenzione (cú@eĩv) delle technai, collocandosi nella lunga serie tradizionale degli inventori divini. Sin qui, dunque, apparentemente nulla di nuovo. Ma nella sua intenzione, le invenzioni tecniche dovrebbero servire a colmare una lacuna per così dire ontogenetica dell'essere umano, la sua originale debolezza e incompiutezza. Perciò esse si definiscono con tutti quei termini che ho voluto citare qui sopra: si tratta fondamentalmente di mezzi, risorse che dànno all'uomo l'illusione, la cieca speranza di potere sfuggire al vincolo di necessità. Il loro stesso inventore, del resto, si trova incatenato, impossibilitato a muoversi e ad agire, a mettere in atto le sue astute risoluzioni contro il potere di Zeus.

Il peccato originale di Prometeo è consistito nel rubare un sapere originariamente non suo, si è impossessato del fuoco rubandolo alla fucina di Efesto, motivo per cui lo stesso Efesto è stato costretto suo malgrado a imprigionare con le sue perfette arti demiurgiche il Titano ribelle. Ma alla prima colpa se ne sono associate presto delle altre, tutte conseguenti, di cui Prometeo a poco a poco, e insieme con lui gli altri personaggi e il pubblico del dramma diventano consapevoli ${ }^{26}$. Altra colpa grave è quella di avere favorito i mortali oltre la giusta misura (Prom.

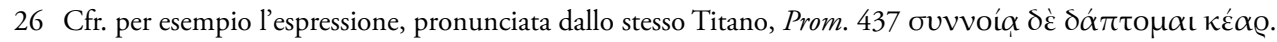




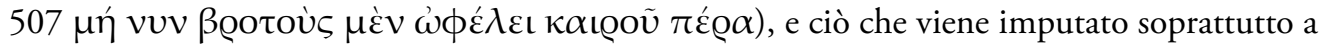
Prometeo è di avere superato il limite, di amare troppo i mortali, come lui stesso osserva, prima

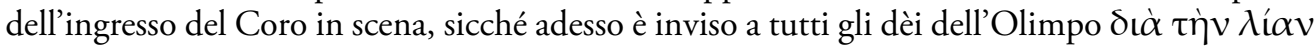

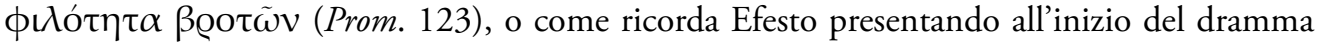
l'indole e l'attitudine del ribelle titano, che ha attribuito onori ai mortali al di là di giustizia

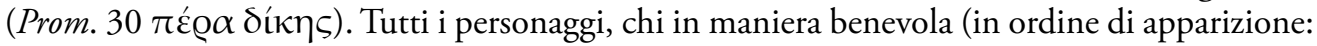
Efesto, il Coro, Oceano), chi invece con toni di deprecazione e di sfida (Kratos al principio, Ermes alla fine), fanno quasi a gara a ricordare a Prometeo il senso della misura, rispetto ad un comportamento del Titano che si lascia riassumere nei termini della tracotanza (v́ß@í̧ $\omega$ : vv.

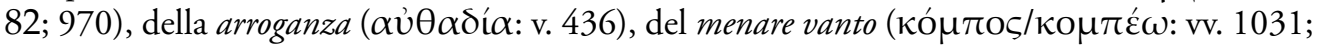

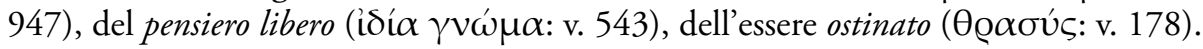

Un tale atteggiamento del protagonista si spiega bene del resto, ove sia inquadrato nella contrapposizione alla figura dell'onnipotente Zeus, l'antagonista sulla scena del dramma eschileo, anch'egli segnato dalla dinamica degli eccessi: come Prometeo ama troppo gli umani, così al contrario Zeus si è distinto per l'eccessiva volontà di sterminio nei loro confronti (Prom.

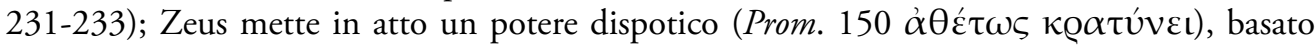
sulla violenza e sulla sopraffazione, sia nei confronti degli dèi che nei confronti degli umani, e alla vicenda di Prometeo fa da contraltare sul piano dei destini umani la vicenda di Io, condannata a vagare ai limiti del mondo abitato sospinta dal tafano inviatole contro da Era, gelosa della passione erotica che la vergine argiva suscitava in Zeus. Ancora, Prometeo rimprovera a Zeus quello stesso comportamento arrogante, da cui aveva inizialmente cercato di difendersi ${ }^{27}$. Sul piano drammatico, la situazione dell' Incatenato è complessivamente quella di un'impasse generale, in cui Prometeo, nonostante le technai, non ha modo di liberarsi dalle catene in cui lo ha costretto Zeus, e a sua volta il padre degli dei è vincolato dal bisogno di conoscere il segreto posseduto da Prometeo, veggente per antonomasia, circa l'identità di chi gli succederà al trono.

Rispetto a questi due estremi di dismisura, il dramma sembra proporre, attraverso la voce degli altri personaggi, un modello improntato alla misura di impronta delfico-apollinea, un richiamo alla vera sapienza tradizionale, non a caso connessa con la pratica divinatoria ${ }^{28}$. E così Efesto, rispondendo alle pressanti richieste provenienti da Kratos di applicare particolare forza nell'operazione di inchiodatura del Titano, gli chiede di non esagerare con gli ordini (Prom. 72

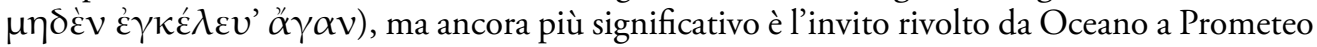

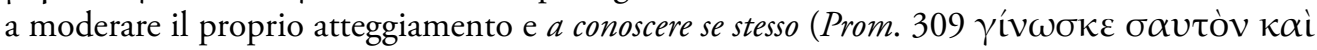

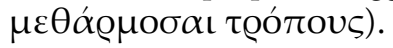

L'atteggiamento arrogante e idiosincratico di Prometeo culmina nell'autocelebrazione con cui il Titano chiosa il catalogo delle technai, rivendicate come una propria invenzione, il cui apprendimento da parte degli uomini passa attraverso l'utilizzo del fuoco - denominato in-

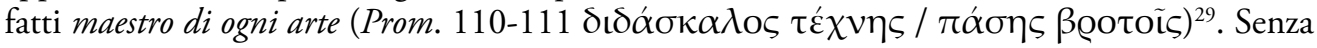
essere squalificate sul piano epistemico, anzi pur assumendo un ruolo semiotico di primo

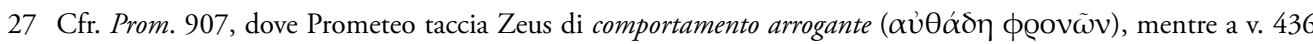
lo stesso Prometeo, anticipando di fatto il rimprovero che il Coro avrebbe di lì a poco mosso nei suoi confronti, esorta le Oceanine a non credere che il suo precedente silenzio sia dovuto a superbia o arroganza $\left(\chi \lambda \mathrm{\iota} \delta \tilde{\eta} \mu \eta \delta^{\prime}\right.$ $\alpha \hat{v} \theta \alpha \delta i ́(1)$, motivo per cui, se non è il caso di ricordare a chi già ne è a conoscenza i benefici arrecati agli dèi, allora istruirà il Coro sulle pene che hanno subìto i mortali da parte di Zeus.

28 Cfr. Di Benedetto 1978, 96 sg.

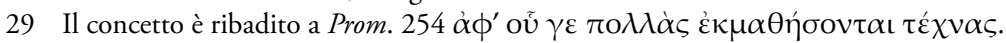


piano nella vita degli uomini, le technai finiscono con l'acquisire nell'accezione prometeica un ruolo di risorse utili per la vita, della cui invenzione Prometeo si può pure vantare, dovendo però riconoscere che a) gli uomini non potranno mai venirgli in aiuto per la loro condizione di mortali ${ }^{30}$, e quindi che la techne nelle loro mani è debole o inadeguata, se confrontata alle possibilità divine, e soprattutto in assoluto che b) la techne è molto più debole della necessità $(\dot{\alpha} v \alpha \dot{\gamma} \gamma \kappa \eta)^{31}$, e che dunque a questa occorre prima o poi soggiacere.

Sul piano delle forme espressive, il vanto di Prometeo dovuto all'invenzione delle technai si realizza nella serie continua di predicati verbali alla prima persona, che si susseguono insisten-

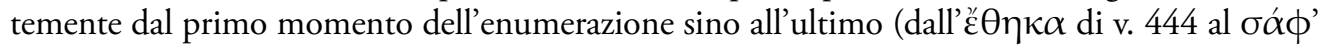
oĩ $\delta \alpha$ di v. 504). Ciò si traduce dal punto di vista del valore sociale delle technai prometeiche nella loro funzione meramente individuale, solipsistica, di mezzi atti al sostentamento, ma inadatti ad una prospettiva politica o latamente societaria. Con questo quadro è coerente la situazione scenica immaginata dal drammaturgo antico, che presenta il Titano umiliato e indifeso, posto ai margini delle terre abitate ${ }^{32}$, in una landa desolata, priva di uomini, in una condizione paradossalmente liminare e senza apparente soluzione. Una situazione, in ultima analisi, in cui, se è vero che la techne tradizionale si è dimostrata asservita alla necessità, le technai prometeiche, figlie del furto del fuoco, espongono chi le possiede, specialmente se umano, alla vertigine del limite.

\section{Postilla critica}

Con le considerazioni che seguono vorrei registrare gli esiti sul testo eschileo di un atteggiamento antitecnico che sembra avere orientato una parte della critica nell'interpretazione di due luoghi segnati dalla presunta presenza del campo lessicale di techne.

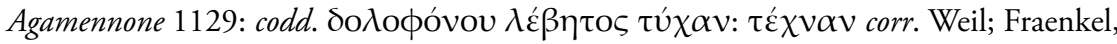
Italie, Löbl probb.

L'episodio è molto noto: Cassandra, la profetessa destinata a non essere mai creduta, ha una orribile visione di morte e di sventura per la reggia di Agamennone, che il Coro interpreta a

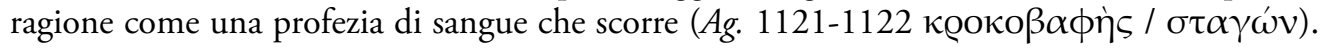
Cassandra evoca quindi l'immagine di un toro che occorre tenere lontano dalla vacca (vv. 1125$1126 \not ̋ \pi \varepsilon \chi \varepsilon \tau \tilde{\alpha} \varsigma \beta$ ßò $/$ iòv $\tau \alpha \tilde{u} \varrho o v)$, chiara allusione alla coppia formata da Agamennone e Clitemestra. Le sue parole si fanno in seguito sempre più chiaramente allusive alla sorte che toccherà ad Agamennone, cadere stecchito, mentre fa il bagno in una vasca piena d'acqua (v. $\left.1128 \pi i ́ \tau v \varepsilon \iota \delta^{\prime}<\dot{\varepsilon} v>\varepsilon^{\prime} v u ́ \delta \varrho \omega \tau \varepsilon u ́ \chi \varepsilon \iota\right)$, sotto i colpi della moglie, avviluppato in un man-

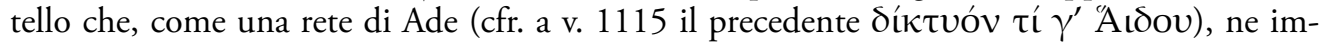

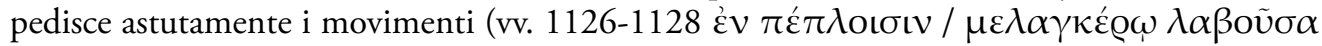
$\mu \eta \chi \alpha v \eta ́ \mu \alpha \tau \iota / \tau u ́ \pi \tau \varepsilon \iota)^{33}$. Perché il Coro non possa fraintendere quanto ha appena annun-

30 Era quanto nelle prime battute del dramma aveva già chiesto provocatoriamente Kratos a Prom. 83-84.

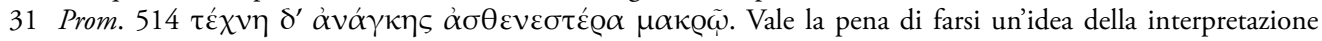
heideggeriana di questa frase messa in discussione da Calame 2016, 49-51.

32 Cfr. Calame 2016, 35 sgg.

33 L'interpretazione semantica e sintattica del passo è piuttosto controversa: alla luce degli scolii, si tratterebbe

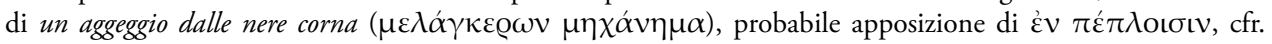
Fraenkel 1962, vol. III, 511 sgg., in part. 515, e la relativa traduzione: with black contrivance of the horned one; la corrispondente traduzione di MoRANi 1987, 457: coi raggiri del nero corno. 
ciato, Cassandra aggiunge: io ti sto raccontando la sorte di un bagno che uccide con inganno (v. 1129). La correzione del tràdito $\tau u ́ \chi \alpha \nu$ in $\tau \hat{\varepsilon} \chi v \alpha \nu$ da parte di H. Weil (Aeschyli quae supersunt tragoediae. Agamemnon, Giessen 1858) ha avuto una certa fortuna; essa nasce dalla memo-

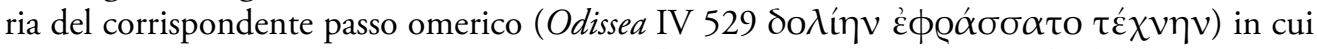
si descrive il sapiente piano, detto appunto techne, con cui Egisto (non si fa alcuna menzione di Clitemestra) prepara la morte di Agamennone, che finisce ucciso come un bue alla greppia;

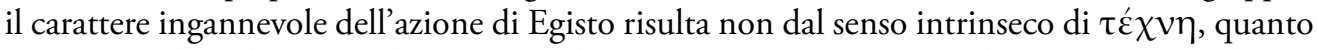
piuttosto dalla relativa attribuzione e dal modo in cui vengono per il resto connotate le sue perfide intenzioni. Fraenkel 1962 nel suo commento ad loc. (vol. III, 516) ritiene la correzione di Weil excellent, dal momento che il senso di $\tau u ́ \chi \alpha$ come "story" sarebbe inappropriato ${ }^{34}$.

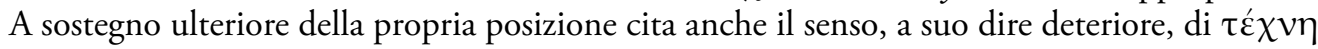

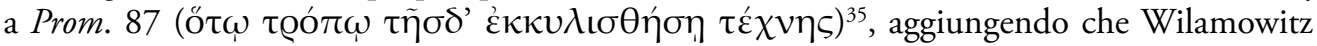
avrebbe segnalato nel commento al passo che alcuni manoscritti riportano la lezione $\tau u ́ \chi \eta \varsigma$ invece di $\tau \dot{\chi} \chi \vee \eta \varsigma$. Come ho avuto modo di illustrare più sopra, il senso del termine nel passo eschileo appena citato rimanda alla grande abilità tecnica di Efesto, e quindi semmai al suo

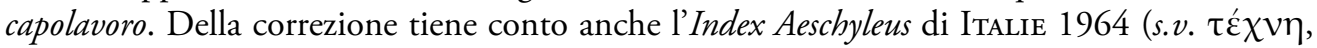
con il significato di “dolus, opus artificiosum”); più recentemente anche LöBL 1997, 74, sembra propenso ad accoglierla, con il senso, appunto negativo, di Täuschung. A questa catena di illazioni critiche hanno infine rimediato due prestigiosi editori del testo eschileo, sia PAGE 1972, che però omette di citare in apparato la correzione, sia da ultimo WEST 1990.

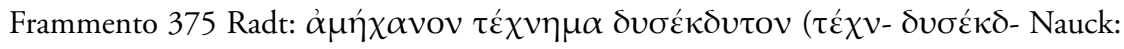
$\tau \varepsilon \dot{\chi} \chi-\delta v \sigma \varepsilon ́ \kappa \lambda-c o d d$.)

Il frammento è contenuto nello scolio ad Eur. Or. 25 ( $\hat{\eta}$, scil. la madre di Oreste, тóotv

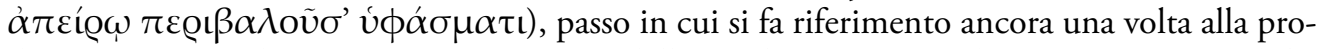
ditoria uccisione di Agamennone per mano della moglie, per mezzo di una veste inestricabile, come si evince anche dalla lettura di alcuni scolii a Hom. Il. I 7 (cfr. RadT 1985, 429). La lezione dei manoscritti $\tau \varepsilon u ́ \chi \eta \mu \alpha$, di per sé assolutamente comprensibile come artefatto (del resto $\tau \varepsilon \dot{u} \chi \omega$ nel significato di "facere" è piuttosto comune nell' uso eschileo) ${ }^{36}$, sulla scorta della valutazione di $A g .1129$, è stata soppiantata dalla correzione $\tau \dot{\varepsilon} \chi \nu \eta \mu \alpha$ proposta da Nauck, piuttosto banale, benché accettabile dal punto di vista semantico e paleografico. I dubbi su $\tau \varepsilon u ́ \chi \eta \mu \alpha$ sembrano derivare dal fatto che si tratterebbe, ove venisse accolto, di un hapax legomenon assoluto, il che non dovrebbe bastare a sancirne l'inautenticità, tanto più che sembra rinviare all'uso eschileo di $\tau \varepsilon \tilde{u} \chi 0 \varsigma$ come vasca da bagno (Ag. 1128). Sia Fraenkel 1962, vol. III, 649 n. 3, sia ITALIE 1964, s.v. $\tau \dot{\varepsilon} \chi \nu \eta \mu \alpha$, accolgono la correzione, e lo stesso fa LöBL 1997, 76, quest'ultimo nella convinzione che non si tratti di un semplice hergestelltes Stück,

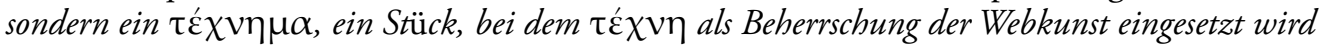

34 Morani 1987 accolgono senza esitazioni la lezione dei mss. traducendo $\tau u ́ \chi \alpha v$ come sorte, che ovviamente non fa riferimento agli eventi precedenti (cfr. FRAENKEL 1962, vol. III, 516: 'the story of a murderous caldron' is hardly suitable here, where the caldron is mentioned only as instrumental to Clytemnestra's plot and not in connexion with any former event), bensì all'esito futuro della vicenda.

35 Come “dolus, opus artificiosum” interpreta l'occorrenza qui del termine Italie 1964, 296, autore dell'Index Aeschyleus, s.v. $\tau \dot{\varepsilon} \chi \vee \eta$.

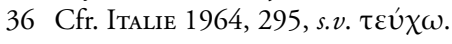


zum Zweck der Überlistung. Ancora una volta, si carica di un significato negativo il vocabolario di techne di per sé, quando invece il termine sembra possedere in Eschilo un'accezione neutra, se non spesso positiva, per cui, semmai si accogliesse $\tau \dot{\varepsilon} \chi \nu \eta \mu \alpha$, esso non potrebbe indicare altro - in quanto nomen rei actae - se non il prodotto finale dell'arte ${ }^{37}$.

\section{Conclusioni}

I drammi di Eschilo rappresentano una testimonianza di primaria importanza per valutare lo sviluppo del concetto di techne tra VI e V sec. a.C., in particolare per quel che riguarda la concezione greca sull'origine delle arti tecniche umane. Alla luce di quanto emerso, Eschilo si fa interprete di una linea di pensiero tradizionale che situa l'origine e il possesso più vero delle technai nelle divinità. La figura di Prometeo non costituisce da questo punto di vista un'eccezione, se non per la significativa differenza che il Titano si appropria di un sapere altrui attraverso il furto con destrezza, e finisce pertanto per opporsi con ostinazione e atteggiamento di sfida al potere appena costituito di Zeus. Dal punto di vista del valore culturale delle technai, il mito prometeico, così come messo in scena nell' Incatenato, rappresenta un importante punto di svolta perché il furto del fuoco comporta l'insegnamento da parte di Prometeo delle tecniche agli uomini. Le technai finiscono così per proiettarsi quanto al loro uso sul piano storico umano, dove assumono nelle intenzioni prometeiche una dimensione sia prettamente utilita-

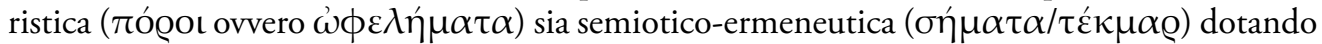
l'uomo di orientamento e di criterio in rapporto all'ambiente che lo contorna. Purtuttavia, la techne nella sua accezione più tradizionale continua a sussistere, caratterizzandosi per il suo statuto demiurgico, rappresentato al meglio dal sapere tecnico-artistico del dio Efesto. Il maggior segno di continuità tra accezione tradizionale delle technai da una parte, e nuova dimensione prometeica dall'altra è dato dall'assoluto ruolo di spicco che nell'opera eschilea spetta alla mantica, indicata come arte che non mente, e che in quanto tale rappresenta, per mezzo del dio delfico Apollo, diretta emanazione del sapere di Zeus. Da questo punto di vista, il possesso della mantica mette in comunicazione l'uomo e il dio, attraverso il rito sacrificale, impedendo all'interprete di pensare che l'essere umano possa prescindere dagli dèi e dal rispetto della

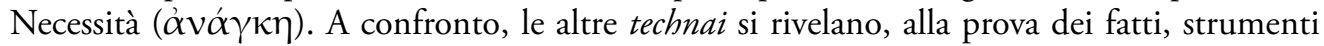
inadatti a liberare l'uomo dal vincolo di necessità, e che lo espongono al rischio continuo della bybris. Sotto questo aspetto, gli uomini si possono dire degni allievi di Prometeo.

Franco Giorgianni

Università degli Studi di Palermo Dipartimento Culture e Società Viale delle Scienze, Edificio 15 franco.giorgianni@unipa.it

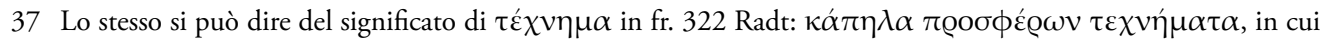
è evidente che a connotare negativamente l'espressione è l'aggettivo $\kappa \alpha ́ \pi \eta \lambda \alpha$, che i lessici etimologici glossano

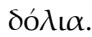




\section{Bibliografia}

Calame 2016

C. Calame, Prometeo genetista. Profitti delle tecniche e metafore della scienza (ed. or. Paris 2010), trad. it., Palermo 2016.

Calame 2017

C. Calame, «À propos des pratiques divinatoires et de la "raison des signes» en Grèce classique ", Mètis N. S. 15 (2017), 393-410.

Cambiano 1991

G. Cambiano, Platone e le tecniche, Bari 1991.

Di Benedetto 1978

V. Di Benedetto, Lideologia del potere e la tragedia greca. Ricerche su Eschilo, Torino 1978.

\section{EDELSTEIN 1987}

L. Edelstein, Lìdea di progresso nell'antichità classica (ed. or. Baltimore 1967), trad. it., Bologna 1987.

Fraenkel 1962

E. Fraenkel (ed.), Aeschylus, Agamemnon. Edited with a Commentary, 3 voll., Oxford 1962 (ristampa).

Gentili, Prato 1979

B. Gentili, G. Prato (edd.), Poetae elegiaci. Testimonia et fragmenta. Pars prior, Leipzig 1979.

\section{GRIFFITH 1983}

M. Griffith (ed.), Aeschylus. Prometheus Bound, Cambridge1983.

ITALIE 1964

G. Italie, Index Aeschyleus. Editio altera correcta et aucta, curavit S. L. Radt, Leiden $1964^{2}$.

LÖBL 1997

R. Löbl, TEXNH - Techne. Untersuchungen zur
Bedeutung dieses Wortes in der Zeit von Homer bis Aristoteles. Band I: Von Homer bis zu den Sophisten, Würzburg 1997.

LÖBL 2003

R. Löbl, TEXNH - Techne. Untersuchungen zur Bedeutung dieses Wortes in der Zeit von Homer bis Aristoteles. Band II: Von den Sophisten bis zu Aristoteles, Würzburg 2003.

Morani 1987

G. e M. Morani (a cura di), Tragedie e frammenti di Eschilo, Torino 1987.

PAge 1972

D. Page (ed.), Aeschyli septem quae supersunt tragoedias edidit, Oxonii 1972

PATtoni 1987

M. P. Pattoni, L'autenticità del Prometeo incatenato di Eschilo, Pisa 1987.

RADT 1985

S. Radt (ed.), Tragicorum Graecorum fragmenta, Vol. 3. Aeschylus, Göttingen 1985.

SÄ̈D 1985

S. Saïd, Sophiste et tyran ou le problème $d u$ Prométhée enchaîne, Paris 1985.

VERNANT 1978

J.-P. Vernant, Mito e pensiero presso i Greci. Studi di psicologia storica (ed. or. Paris 1971), trad. it., Torino $1978^{2}$.

\section{West 1990}

M. L. West (ed.), Aeschyli tragoediae cum incerti poetae Prometheo, Stutgardiae 1990. 